\title{
Sequential Point Estimation of Location Parameter in Location-Scale Family of Non-Regular Distributions
}

\author{
Ken-ichi Koike \\ Institute of Mathematics, University of Tsukuba, Tsukuba, Ibaraki, Japan
}

\begin{abstract}
In this paper, we consider sequential estimation of the location parameter based on the midrange in the presence of unknown scale parameter when the underlying distribution has a bounded support. The estimation is done under squared loss plus cost of sampling. Stopping rules based on the range are proposed and they are shown to be asymptotically efficient. The risks of the sequential procedures are compared with the Robbins' sequential estimation procedure based on the sample mean. The formers are shown to be asymptotically more efficient than the latter in the sense of the sample size when the density function changes sharply at the end points of the support. Koike (2007) observed a similar asymptotic superiority of the sequential estimation procedure based on the midrange in the sequential interval estimation procedure under the same condition.
\end{abstract}

Keywords: Extreme value; Non-regular case; Robbins' procedure; Sequential point estimation.

Subject Classifications: 62L12; 62F10.

\section{INTRODUCTION}

Suppose that $X_{1}, X_{2}, \ldots$ are independent and identically distributed (i.i.d.) with $E\left(X_{1}\right)=\mu$ and $V\left(X_{1}\right)=\sigma^{2}>0$. We consider the estimation problem of $\mu$ under the squared loss plus cost. If $\mu$ is estimated by the sample mean $\bar{X}_{n}=\sum_{i=1}^{n} X_{i} / n$, then the risk is given by

Address correspondence to Ken-ichi Koike, Institute of Mathematics, University of Tsukuba, 1-1-1 Tennodai, Tsukuba, Ibaraki 305-8571, Japan; Fax: 81-29-853-6501; E-mail: koike@math.tsukuba.ac.jp 


$$
r_{n}^{\prime}:=E\left(\bar{X}_{n}-\mu\right)^{2}+d n=\sigma^{2} / n+d n,
$$

where $d(>0)$ is the cost per observation. If $\sigma$ is known, then the risk is minimized at the integer closest to $n_{d}^{\prime}:=\sigma / \sqrt{d}$. For simplicity, we will assume that $n_{d}^{\prime}$ is an integer. Then the minimized risk is $r_{n_{d}^{\prime}}^{\prime}=2 d n_{d}^{\prime}=$ $2 \sqrt{d} \sigma$. However, unless $\sigma$ is known, one can not attain this risk with a nonsequential procedure. For normal random variables, Robbins (1959) proposed the following stopping rule:

$$
T_{d}^{\prime}:=\left\{n \geq m_{d}^{\prime} \mid n^{2} \geq v_{n} / d\right\} \quad\left(v_{n}:=\frac{1}{n-1} \sum_{i=1}^{n}\left(X_{i}-\bar{X}_{n}\right)^{2}\right),
$$

where $m_{d}^{\prime}$ is the initial sample size. Ghosh and Mukhopadhyay (1979) and Chow and $\mathrm{Yu}$ (1981) showed that, under some conditions, the sequential estimation procedure $\left(T_{d}^{\prime}, \bar{X}_{T_{d}^{\prime}}\right)$ is asymptotically risk efficient, that is, $r_{T_{d}^{\prime}}^{\prime} / r_{n_{d}^{\prime}}^{\prime} \rightarrow$ 1 as $d \rightarrow 0$, without normality assumption. Chow and Martinsek (1982) showed that $\left(T_{d}^{\prime}, \bar{X}_{T_{d}^{\prime}}\right)$ has bounded regret in the sense $\left(r_{T_{d}^{\prime}}^{\prime}-r_{n_{d}^{(1)}}^{\prime}\right) / d=O(1)$. For another major reference, see Ghosh et al. (1997).

As a typical non-regular case, some sequential estimation procedures are obtained for the uniform distribution by Akahira and Koike (2005), Akahira and Takeuchi (2003), Chaturvedi et al. (2001), Govindarajulu (1997), Mukhopadhyay et al. (1983), Mukhopadhyay (1987) and Wald (1950) among others. Basawa et al. (1990) also discusses non-regular cases for the bounded risk point estimation under a general setting.

In Section 2, we consider sequential estimation of the location parameter based on the midrange in the presence of unknown scale parameter when the underlying distribution has a bounded support. The estimation is done under squared loss plus cost of sampling. In Subsection 2.1, we consider the case when the underlying density function has positive limit values at the end points of the support. A stopping rule based on the range is proposed and it is shown to be asymptotically efficient. The risks of the sequential procedures are compared with the Robbins' sequential estimation procedure based on the sample mean. The former is shown to be asymptotically more efficient than the latter in the sense of the sample size. In Subsection 2.2, we consider a case when the underlying density function converges to 0 at the end points of the support. We investigate a sequential estimation procedure based on the range, compare with the Robbins' sequential estimation procedure and show an asymptotic superiority of the estimation procedure 
based on the midrange to the Robbins' procedure when the density function changes sharply at the end points of the support.

Koike (2007) observed a similar asymptotic superiority of the sequential estimation procedure based on the midrange in the sequential interval estimation procedure under the same condition.

\section{SEQUENTIAL ESTIMATION PROCEDURES}

In this section we consider sequential estimation procedures for two cases below.

Let $Z_{1}, Z_{2}, \ldots$ be a sequence of i.i.d. random variables according to the density function $f_{0}(x-\theta)\left(\theta \in \mathbb{R}^{1}\right)$ with respect to the Lebesgue measure. We assume throughout the paper that $f_{0}(x)$ has a bounded support $(-a, a)$ $(a>0)$, i.e., $f_{0}(x)>0$ for $-a<x<a$, and $f_{0}(x)=0$ otherwise, and is twice continuously differentiable in $(-a, a)$. Note that if the support of $f_{0}$ is $(-a, b)(a \neq b)$, then the normalized midrange does not converge to $\theta$ in probability as $n \rightarrow \infty$.

We consider the following two cases as non-regular distribution.

(A1) $f_{0}(x)$ satisfies

$$
\left\{\begin{array}{l}
\lim _{x \rightarrow-a+0} f_{0}(x)=c_{1}(>0), \quad \lim _{x \rightarrow a-0} f_{0}(x)=c_{2}(>0), \\
\lim _{x \rightarrow-a+0} f_{0}^{\prime}(x)=h_{1}, \quad \lim _{x \rightarrow a-0} f_{0}^{\prime}(x)=h_{2},
\end{array}\right.
$$

where $c_{1}, c_{2}, h_{1}$ and $h_{2}$ are some constants.

(A2) $f_{0}(x)$ satisfies

$$
\lim _{x \rightarrow-a+0}(x+a)^{-\gamma} f_{0}(x)=g_{1}, \quad \lim _{x \rightarrow a-0}(a-x)^{-\gamma} f_{0}(x)=g_{2},
$$

where $\gamma, g_{1}$ and $g_{2}$ are some positive constants.

In (A2), if the converging order $\gamma$ 's are different, then the normalized midrange does not converge to $\theta$ in probability as $n \rightarrow \infty$. Note that $f_{0}(x)$ satisfying (A2) converges to 0 with the order of $(x+a)^{\gamma}$ and $|x-a|^{\gamma}$ as $x \rightarrow-a+0$ and $x \rightarrow a-0$, respectively. So, the density changes sharply at the end points of the support if $0<\gamma<1$ and changes smoothly if $\gamma>1$. These conditions are essentially the same as those in Akahira (1975a, b), Akahira and Takeuchi (1981, p. 31; 1995, pp. 81, 148) and Koike (2007).

We consider the cases of (A1) and (A2) in Subsections 2.1 and 2.2, respectively. 


\subsection{Estimation Procedure for (A1)}

In this subsection we treat the case of (A1). At first, we consider the asymptotic distribution of the extreme values in a similar way to Akahira and Takeuchi (1995) and Koike (2007).

Under (A1), by putting $Z_{(1)}:=\min _{1 \leq i \leq n} Z_{i}, Z_{(n)}:=\max _{1 \leq i \leq n} Z_{i}, U:=$ $n\left(Z_{(1)}+a-\theta\right)$ and $V:=n\left(Z_{(n)}-a-\theta\right)$, the joint density of $(U, V)$ is expanded as

$$
\begin{aligned}
& f_{U, V}^{(n)}(u, v) \\
& =\left\{\begin{array}{rr}
\exp \left\{-\left(u c_{1}-v c_{2}\right)\right\}\left[c_{1} c_{2}+\frac{1}{n}\left\{-c_{1} c_{2}+c_{1} c_{2}\left(2\left(u c_{1}-v c_{2}\right)\right.\right.\right. \\
\left.\left.\left.-\left(\frac{h_{1} u^{2}}{2}-\frac{h_{2} v^{2}}{2}\right)-\frac{1}{2}\left(u c_{1}-v c_{2}\right)^{2}\right)+h_{1} u c_{2}+h_{2} v c_{1}\right\}\right] \\
+o\left(\frac{1}{n}\right) & (v<0<u), \\
0 & \text { (otherwise) }
\end{array}\right.
\end{aligned}
$$

from Koike (2007).

Now, we consider the location-scale parameter family of distributions with a bounded support $(\theta-\xi a, \theta+\xi a)$. Suppose that $X_{1}, X_{2}, \ldots$ is a sequence of i.i.d. random variables with the density $(1 / \xi) f_{0}((x-\theta) / \xi)$, where $\theta \in \mathbb{R}$ and $\xi>0$. Put $Y_{i}:=\left(X_{i}-\theta\right) / \xi$ for each $i=1,2, \ldots$, and $Y_{(1)}:=\min _{1 \leq i \leq n} Y_{i}$, $Y_{(n)}:=\max _{1 \leq i \leq n} Y_{i}$. Letting $S:=n\left(Y_{(1)}+Y_{(n)}\right) / 2$ and $T:=n\left(Y_{(1)}-Y_{(n)}+\right.$ $2 a) / 2$, we have the asymptotic joint density of $(S, T)$

$$
f_{S, T}(s, t)= \begin{cases}2 c_{1} c_{2} \exp \left\{-\left(c_{1}-c_{2}\right) s-\left(c_{1}+c_{2}\right) t\right\} & (t>|s|), \\ 0 & \text { (otherwise) }\end{cases}
$$

from (2.1). The asymptotic density of $S$ is given by

$$
f_{S}(s)= \begin{cases}K e^{-2 c_{1} s} & (s \geq 0), \\ K e^{2 c_{2} s} & (s<0),\end{cases}
$$

where $K=2 c_{1} c_{2} /\left(c_{1}+c_{2}\right)$. So, the asymptotic expectations of $S$ and $S^{2}$ are

$$
\begin{aligned}
& E(S) \approx K\left\{\int_{0}^{\infty} s e^{-2 c_{1} s} d s+\int_{-\infty}^{0} s e^{2 c_{2} s} d s\right\}=\frac{c_{2}-c_{1}}{2 c_{1} c_{2}} \\
& E\left(S^{2}\right) \approx K\left\{\int_{0}^{\infty} s^{2} e^{-2 c_{1} s} d s+\int_{-\infty}^{0} s^{2} e^{2 c_{2} s} d s\right\}=\frac{c_{2}^{2}-c_{1} c_{2}+c_{1}^{2}}{2\left(c_{1} c_{2}\right)^{2}} .
\end{aligned}
$$


So, we may assume the following condition.

(B1) There exists a positive constant $A$ satisfying $E\left(S^{2}\right) \rightarrow A$ as $n \rightarrow \infty$.

Concerning this assumption, we have the following lemma.

Lemma 2.1. (B1) and $E\left(S^{4}\right)=O(1)$ hold under (A1).

Proof. At first, we show (B1). From Fatou's lemma and (2.2),

$$
\liminf _{n \rightarrow \infty} E\left(S^{2}\right) \geq \frac{c_{2}^{2}-c_{1} c_{2}+c_{1}^{2}}{2\left(c_{1} c_{2}\right)^{2}} .
$$

Since $S=n\left(Y_{(1)}+Y_{(n)}\right) / 2$ and $0 \leq E\left\{\left(Y_{(1)}+Y_{(n)}\right)^{2}\right\} \leq 2\left[E\left\{\left(Y_{(1)}+a\right)^{2}\right\}+\right.$ $\left.E\left\{\left(Y_{(n)}-a\right)^{2}\right\}\right]$, it suffices to show $E\left\{\left(Y_{(1)}+a\right)^{2}\right\}=O\left(n^{-2}\right)$ and $E\left\{\left(Y_{(n)}-\right.\right.$ $\left.a)^{2}\right\}=O\left(n^{-2}\right)$. The density of $Y_{(1)}$ is given by

$$
f_{Y_{(1)}}(x)=n\{1-F(x)\}^{n-1} f_{0}(x),
$$

where $F$ is the distribution function of $Y_{1}$, hence,

$$
\begin{aligned}
E\left\{\left(Y_{(1)}+a\right)^{2}\right\} & =\left(\int_{-a}^{-a+\epsilon}+\int_{-a+\epsilon}^{a}\right)(x+a)^{2} n\{1-F(x)\}^{n-1} f_{0}(x) d x \\
& =: I_{1}+I_{2} \quad \text { (say). }
\end{aligned}
$$

Putting $y=n(x+a)$, we have, for a sufficiently small $\epsilon>0$,

$$
\begin{aligned}
I_{1} & =\int_{0}^{n \epsilon}\left(n^{-1} y\right)^{2} n\left\{1-F\left(-a+n^{-1} y\right)\right\}^{n-1} f_{0}\left(-a+n^{-1} y\right) n^{-1} d y \\
& =\int_{0}^{n \epsilon} n^{-2} y^{2} \exp (-c y)\left\{1-\frac{h_{1}}{2} y^{2} n^{-1}+o\left(n^{-1}\right)\right\}\left\{c+h_{1} n^{-1}+o\left(n^{-1}\right)\right\} d y \\
& =n^{-2} \int_{0}^{n \epsilon} y^{2} \exp (-c y)\left\{c+n^{-1}\left(-\frac{c h_{1}}{2} y^{2}+h_{1} y\right)+o\left(n^{-1}\right)\right\} d y \\
& \leq C n^{-2}
\end{aligned}
$$

where the second equality follows from the expansion of $f_{0}(x)$, and $C$ is some positive constant. On the other hand, since

$$
\begin{aligned}
I_{2} & =\int_{-a+\epsilon}^{a}(x+a)^{2} n\{1-F(x)\}^{n-1} f_{0}(x) d x \\
& \leq n\{1-F(-a+\epsilon)\}^{n-1} \int_{-a+\epsilon}^{a}(x+a)^{2} n f_{0}(x) d x \\
& =n\{1-F(-a+\epsilon)\}^{n-1} E\left\{\left(Y_{1}+a\right)^{2}\right\}=O\left(n^{-2}\right),
\end{aligned}
$$

we have $E\left\{\left(Y_{(1)}+a\right)^{2}\right\}=O\left(n^{-2}\right)$. 
In a similar way to the above, we have $E\left\{\left(Y_{(n)}-a\right)^{2}\right\}=O\left(n^{-2}\right)$, thus $E\left(S^{2}\right)=O(1)$. And we can show $E\left(S^{4}\right)=O(1)$ similarly. Therefore we have the desired result.

If the population distribution is the uniform distribution $U(-1,1)$, then the constant $A$ is calculated exactly. In fact, an easy computation yields

$$
\begin{aligned}
& E\left(S^{2}\right)=\frac{2 n^{2}}{(n+1)(n+2)} \rightarrow 2, \\
& E\left(S^{4}\right)=\frac{24 n^{4}}{(n+1)(n+2)(n+3)(n+4)} \rightarrow 24 .
\end{aligned}
$$

If $\theta$ is estimated by the midrange $M_{n}=\left(X_{(1)}+X_{(n)}\right) / 2$, then the risk is given by

$$
r_{n}:=E\left(M_{n}-\theta\right)^{2}+d n,
$$

where $d(>0)$ is the cost per observation. From $S=n\left(M_{n}-\theta\right) / \xi$ and (A2), $r_{n}$ is approximated by $\left(A \xi^{2} / n^{2}\right)+d n$, which is minimized at the integer closest to $n=n_{d}^{(1)}:=\left(2 A \xi^{2} / d\right)^{1 / 3}$ and the minimized value is $r_{n_{d}^{(1)}}=3\left(A \xi^{2} d^{2}\right)^{1 / 3} / 2^{2 / 3}$. However, unless $\xi$ is known, one can not attain this risk with a non-sequential procedure. Since the range $R_{n}:=X_{(n)}-X_{(1)}$ converges to $2 a \xi$ almost surely as $n \rightarrow \infty$, therefore we consider the following stopping rule:

$$
T_{d}^{(1)}:=\inf \left\{n \geq m_{d}^{(1)} \mid n^{3} \geq A R_{n}^{2} /\left(2 a^{2} d\right)\right\},
$$

where $m_{d}^{(1)}$ is the initial sample size with $d^{-l} \leq m_{d}^{(1)}=o\left(d^{-1 / 3}\right)(0<l<1 / 3)$. Then we have the following theorem.

Theorem 2.1. Under the conditions (A1) and (B1), as $d \rightarrow 0$, we have
(i) $T_{d}^{(1)} / n_{d}^{(1)} \stackrel{\text { a.s. }}{\rightarrow} 1$,
(ii) $E\left(T_{d}^{(1)}\right) / n_{d}^{(1)} \rightarrow 1$
(iii) $r_{T_{d}^{(1)}} / r_{n_{d}^{(1)}} \rightarrow 1$.

Proof. At first, we note that

$$
m_{d}^{(1)} \leq T_{d}^{(1)} \leq n_{d}^{(1)}+1 \quad \text { with probability } 1 .
$$

In fact, since $0 \leq R_{n} \leq 2 a \xi$ with probability 1 , it holds $0 \leq\left(A R_{n}^{2} /\left(2 a^{2} d\right)\right)^{1 / 3}$ $\leq\left(2 A \xi^{2} / d\right)^{1 / 3}$ with probability 1. Hence, $n>\left(A R_{n}^{2} /\left(2 a^{2} d\right)\right)^{1 / 3}$ for $n$ satisfying $n>\left(2 A \xi^{2} / d\right)^{1 / 3}$. Therefore $(2.3)$ holds. Since $T_{d}^{(1)} \stackrel{\text { a.s. }}{\rightarrow} \infty$ and $R_{n} \stackrel{\text { a.s. }}{\rightarrow} 2 a \xi$, $R_{T_{d}^{(1)}} \stackrel{\text { a.s. }}{\rightarrow} 2 a \xi$. By the definition of $T_{d}^{(1)}$,

$$
\left(\frac{A R_{T_{d}^{(1)}}^{2}}{2 a^{2} d}\right)^{1 / 3} \leq T_{d}^{(1)}<m_{d}^{(1)}+\left(\frac{A R_{T_{d}^{(1)}-1}^{2}}{2 a^{2} d}\right)^{1 / 3} .
$$


Dividing this by $n_{d}^{(1)}$, we have (i) as $d \rightarrow 0$ since $d^{-l} \leq m_{d}^{(1)}=o\left(d^{-1 / 3}\right)$. To prove (ii), we have from (i) that

$$
\liminf _{d \rightarrow 0} E\left(T_{d}^{(1)} / n_{d}^{(1)}\right) \geq 1
$$

by Fatou's lemma. On the other hand, by (2.3),

$$
\frac{E\left(T_{d}^{(1)}\right)}{n_{d}^{(1)}} \leq \frac{\left(2 A \xi^{2} / d\right)^{1 / 3}+1}{\left(2 A \xi^{2} / d\right)^{1 / 3}} \rightarrow 1 \quad(d \rightarrow 0)
$$

hence $E\left(T_{d}^{(1)}\right) / n_{d}^{(1)} \rightarrow 1$ as $d \rightarrow 0$. So, we have (ii).

To prove (iii), we may assume $\theta=0$ without loss of generality, since $M_{n}$ is location equivariant. Putting $S_{k, n}:=(k+n) M_{k+n}-k M_{k}(k \geq 0, n \geq 1)$, we have by Minkowski's inequality, that

$$
\begin{aligned}
0 & \leq\left(E\left|S_{k, n}\right|^{4}\right)^{1 / 4}=\left(E\left|(k+n) M_{k+n}-k M_{k}\right|^{4}\right)^{1 / 4} \\
& \leq\left(E\left|(k+n) M_{k+n}\right|^{4}\right)^{1 / 4}+\left(E\left|k M_{k}\right|^{4}\right)^{1 / 4}=O(1)
\end{aligned}
$$

from Lemma 2.1. Taking $\eta$ and $\lambda$ satisfying $0<\lambda<\left(A \xi^{2}\right)^{1 / 3}<\eta$, we have $P\left((d / 2)^{1 / 3} T_{d}^{(1)} \geq \eta\right) \rightarrow 0$ as $d \rightarrow 0$ from (i). By (2.4) and Theorem B of Serfling (1980),

$$
E \max _{1 \leq i \leq n}\left|S_{k, i}\right|^{4}=O(1) \quad \text { for } k \geq k_{0}, n \geq 1 .
$$

Since $T_{d}^{(1)} \geq m_{d}^{(1)}$ with probability 1 ,

$$
\begin{aligned}
& \eta^{-2}(d / 2)^{2 / 3} E\left\{\left(T_{d}^{(1)} M_{T_{d}^{(1)}}\right)^{2} I\left(\lambda \leq(d / 2)^{1 / 3} T_{d}^{(1)} \leq \eta\right)\right\} \\
\leq & E\left(M_{T_{d}^{(1)}}^{2}\right) \\
\leq & E\left\{M_{T_{d}^{(1)}}^{2} I\left(T_{d}^{(1)} \leq \lambda(2 / d)^{1 / 3}\right)\right\} \\
& \quad+\lambda^{-2}(d / 2)^{2 / 3} E\left\{\left(T_{d}^{(1)} M_{T_{d}^{(1)}}\right)^{2} I\left(\lambda \leq(d / 2)^{1 / 3} T_{d}^{(1)} \leq \eta\right)\right\} \\
& \quad+E\left\{M_{T_{d}^{(1)}}^{2} I\left(T_{d}^{(1)} \geq \eta(2 / d)^{1 / 3}\right)\right\},
\end{aligned}
$$

where $I(A)$ is the indicator function of an event $A$. By Schwarz's inequality and (2.5),

$$
\begin{aligned}
& E\left\{M_{T_{d}^{(1)}}^{2} I\left(T_{d}^{(1)} \geq \eta(2 / d)^{1 / 3}\right)\right\} \\
& \leq \eta^{-2}(d / 2)^{2 / 3} \sum_{j=0}^{\infty} 2^{-2 j}\left[E\left\{\max _{2^{j} \eta(2 / d)^{1 / 3} \leq n \leq 2^{j+1} \eta(2 / d)^{1 / 3}}\left|n M_{n}\right|^{4}\right\}\right]^{1 / 2}
\end{aligned}
$$




$$
\begin{aligned}
& \cdot\left[P\left\{2^{j} \eta(2 / d)^{1 / 3} \leq T_{d}^{(1)} \leq 2^{j+1} \eta(2 / d)^{1 / 3}\right\}\right]^{1 / 2} \\
= & o\left(d^{2 / 3} \sum_{j=0}^{\infty} 2^{-2 j} 2^{j} d^{-1 / 3}\right)=o\left(d^{1 / 3}\right)
\end{aligned}
$$

since $P\left(T_{d}^{(1)} \geq \eta(2 / d)^{1 / 3}\right) \rightarrow 0$ as $d \rightarrow 0$. For an $\varepsilon>0$ satisfying $\lambda^{3}<$ $\left(A \xi^{2}\right)-\varepsilon$,

$$
\begin{aligned}
& P\left\{T_{d}^{(1)} \leq \lambda(2 / d)^{1 / 3}\right\} \\
\leq & P\left\{\lambda(2 / d)^{1 / 3} \geq\left(\frac{A R_{n}}{2 a^{2} d}\right)^{1 / 3} \text { for some } m_{d}^{(1)} \leq n \leq \lambda(2 / d)^{1 / 3}\right\} \\
= & P\left\{\lambda^{3} \geq \frac{A R_{n}^{2}}{4 a^{2}} \text { for some } m_{d}^{(1)} \leq n \leq \lambda(2 / d)^{1 / 3}\right\} \\
\leq & P\left\{1-\left(\frac{R_{n}}{2 a \xi}\right)^{2}>\frac{\varepsilon}{A \xi^{2}} \text { for some } m_{d}^{(1)} \leq n \leq \lambda(2 / d)^{1 / 3}\right\} \\
= & P\left\{0 \leq \frac{R_{n}}{2 a \xi}<\sqrt{1-\frac{\varepsilon}{A \xi^{2}}} \text { for some } m_{d}^{(1)} \leq n \leq \lambda(2 / d)^{1 / 3}\right\} \\
\leq & \left.P\left(0 \leq \frac{R_{m_{d}^{(1)}}}{2 a \xi}<\sqrt{1-\frac{\varepsilon}{A \xi^{2}}}\right) \quad \text { (by the monotonicity of } R_{n} \text { w.r.t. } n\right)
\end{aligned}
$$

$$
=O\left(\alpha^{m_{d}^{(1)}}\right) \text {, }
$$

where $\alpha \in(0,1)$ is a constant. (2.7) follows from the estimation of the probability of the event $\left\{R_{n} \leq l\right\}(l>0)$. In fact, putting $R_{n}^{\prime}=Z_{(n)}-Z_{(1)}$, we have

$$
\begin{aligned}
P\left(R_{n}^{\prime} \leq l\right) & =P\left(Z_{(n)}-Z_{(1)} \leq l\right) \leq P\left\{\left\{Z_{(1)} \geq-l / 2\right\} \cup\left\{Z_{(n)} \leq l / 2\right\}\right\} \\
& \leq P\left\{Z_{(1)} \geq-l / 2\right\}+P\left\{Z_{(n)} \leq l / 2\right\} .
\end{aligned}
$$

Let $G$ be the distribution function of $Z_{1}$. Since $P\left\{Z_{(1)} \geq-l / 2\right\}=\{1-$ $G(-l / 2)\}^{n}$

$=\alpha_{1}^{n}$ (say) and $P\left\{Z_{(n)} \leq l / 2\right\}=G^{n}(l / 2)=\alpha_{2}^{n}$ (say), $P\left(R_{n}^{\prime} \leq l\right) \leq \alpha_{1}^{n}+\alpha_{2}^{n}$, hence (2.7) holds. By Schwarz's inequality and (2.7),

$$
\begin{aligned}
& E\left\{M_{T_{d}^{(1)}}^{2} I\left(T_{d}^{(1)} \leq \lambda(2 / d)^{1 / 3}\right)\right\} \\
\leq & \left\{E\left|M_{T_{d}^{(1)}}\right|^{4}\right\}^{1 / 2}\left[P\left\{T_{d}^{(1)} \leq \lambda(2 / d)^{1 / 3}\right\}\right]^{1 / 2} \\
\leq & \sum_{j: 2^{j} \geq m_{d}^{(1)}} 2^{-2 j}\left\{E\left(\max _{2^{j} \leq n \leq 2^{j+1}}\left|n M_{n}\right|^{4}\right)\right\}^{1 / 2}\left[P\left\{T_{d}^{(1)} \leq \lambda(2 / d)^{1 / 3}\right\}\right]^{1 / 2}
\end{aligned}
$$




$$
=D \sum_{j: 2^{j} \geq m_{d}^{(1)}} 2^{-2 j}\left(O\left(\alpha^{m_{d}^{(1)}}\right)\right)^{1 / 2}=O\left(m_{d}^{(1)^{-1}} \alpha^{m_{d}^{(1)} / 2}\right),
$$

where $D$ is some constant. On the other hand, since $\left|a^{2}-b^{2}\right| \leq|a-b|^{2}+$ $2|b||a-b|$ for $a, b \in \mathbb{R}$,

$$
\begin{aligned}
& \mid E\left\{\left(T_{d}^{(1)} M_{T_{d}^{(1)}}\right)^{2} I\left(\lambda \leq(d / 2)^{1 / 3} T_{d}^{(1)} \leq \eta\right)\right\} \\
& -E\left\{\left(\left[\lambda(2 / d)^{1 / 3}\right] M_{\left[\lambda(2 / d)^{1 / 3}\right]}\right)^{2}\right\} \mid \\
& \leq E\left\{\max _{\lambda(2 / d)^{1 / 3} \leq n \leq \eta(2 / d)^{1 / 3}}\left|\left(n M_{n}\right)^{2}-\left(\left[\lambda(2 / d)^{1 / 3}\right] M_{\left[\lambda(2 / d)^{1 / 3}\right]}\right)^{2}\right|\right\} \\
& +E\left[\left(\left[\lambda(2 / d)^{1 / 3}\right] M_{\left[\lambda(2 / d)^{1 / 3}\right]}\right)^{2}\right. \\
& \left.\quad \cdot\left\{I\left((d / 2)^{1 / 3} T_{d}^{(1)}<\lambda\right)+I\left((d / 2)^{1 / 3} T_{d}^{(1)}>\eta\right)\right\}\right] \\
& \leq\left\{E\left(\max _{\lambda(2 / d)^{1 / 3} \leq n \leq \eta(2 / d)^{1 / 3}}\left|n M_{n}-\left[\lambda(2 / d)^{1 / 3}\right] M_{\left[\lambda(2 / d)^{1 / 3}\right]}\right|^{4}\right)\right\}^{1 / 2} \\
& +2\left[E\left\{\left(\left[\lambda(2 / d)^{1 / 3}\right] M_{\left[\lambda(2 / d)^{1 / 3}\right]}\right)^{2}\right\}\right]^{1 / 2} \\
& \quad \cdot\left\{E\left(\max _{\lambda(2 / d)^{1 / 3} \leq n \leq \eta(2 / d)^{1 / 3}}\left|n M_{n}-\left[\lambda(2 / d)^{1 / 3}\right] M_{\left[\lambda(2 / d)^{1 / 3}\right]}\right|^{4}\right)\right\}^{1 / 4} \\
& +\left\{E\left|\left[\lambda(2 / d)^{1 / 3}\right] M_{\left[\lambda(2 / d)^{1 / 3}\right]}\right|^{4}\right\}^{2} \\
& \quad \cdot\left\{P^{1 / 2}\left((d / 2)^{1 / 3} T_{d}^{(1)}<\lambda\right)+P^{1 / 2}\left((d / 2)^{1 / 3} T_{d}^{(1)}>\eta\right)\right\}
\end{aligned}
$$

from Schwarz's inequality. Therefore, since $E\left(\left[\lambda(2 / d)^{1 / 3}\right] M_{\left[\lambda(2 / d)^{1 / 3}\right]}\right)^{2} \sim A \xi^{2}$ as $d \rightarrow 0$, and $\eta$ and $\lambda$ can be taken arbitrary close to $\left(A \xi^{2}\right)^{1 / 3}$,

$$
E\left(\hat{\theta}_{T_{d}^{(1)}}-\theta\right)^{2} \sim\left(A \xi^{2}\right)^{1 / 3}(d / 2)^{2 / 3} .
$$

By (ii) and (2.8), we have (iii).

Remark 2.1. The above proof of (iii) is basically based on that of Theorem 1 of Lai (1996), which shows that, in a general setting, a sequential estimation procedure based on a $\sqrt{n}$-consistent estimate is risk-efficient, whereas we treat a sequential estimation procedure based on an $n$-consistent estimate.

From Theorem 2.1 and Chow and $\mathrm{Yu}$ (1981), as $d \rightarrow 0$,

$$
\frac{r_{T_{d}^{(1)}}}{r_{T_{d}^{\prime}}^{\prime}} \approx \frac{3\left(A \xi^{2} d^{2}\right)^{1 / 3} / 2^{2 / 3}}{2 \sqrt{d} \sigma} \rightarrow 0,
$$


where $\sigma^{2}=V\left(X_{1}\right)$. So, the estimation procedure $\left(T_{d}^{(1)}, M_{T_{d}^{(1)}}\right)$ is asymptotically better than $\left(T_{d}^{\prime}, \bar{X}_{T_{d}^{\prime}}\right)$. A similar phenomenon that the sequential interval estimation procedure based on the midrange is asymptotically better than the sample mean can be found in Koike (2007).

\subsection{Estimation Procedure for (A2)}

In this subsection, we consider the case of (A2).

By putting $U^{\prime}:=n^{1 /(\gamma+1)}\left(Z_{(1)}+a-\theta\right)$ and $V^{\prime}:=n^{1 /(\gamma+1)}\left(Z_{(n)}-a-\theta\right)$ with $Z_{(1)}=\min _{1 \leq i \leq n} Z_{i}$ and $Z_{(n)}=\max _{1 \leq i \leq n} Z_{i}$, under the condition (A2), the joint density $f_{U^{\prime}, V^{\prime}}^{(n)}(u, v)$ of $\left(U^{\prime}, V^{\prime}\right)$ satisfies

$$
f_{U^{\prime}, V^{\prime}}^{(n)}(u, v) \rightarrow \begin{cases}g_{1} g_{2}(-u v)^{\gamma} \exp \left\{-\frac{g_{2}}{\gamma+1}(-v)^{\gamma+1}-\frac{g_{1}}{\gamma+1} u^{\gamma+1}\right\} & (v<0<u), \\ 0 & \text { (otherwise) }\end{cases}
$$

as $n \rightarrow \infty$ (Koike (2007)).

Suppose that $X_{1}, X_{2}, \ldots$ is a sequence of i.i.d. random variables with the density $(1 / \xi) f_{0}((x-\theta) / \xi)$, where $\theta \in \mathbb{R}$ and $\xi>0$. Put $Y_{i}:=\left(X_{i}-\theta\right) / \xi$ for each $i=1,2, \ldots$, and $Y_{(1)}:=\min _{1 \leq i \leq n} Y_{i}, Y_{(n)}:=\max _{1 \leq i \leq n} Y_{i}$.

Letting $S^{\prime}:=n^{1 /(\gamma+1)}\left(Y_{(1)}+Y_{(n)}\right) / 2$ and $T^{\prime}:=n^{1 /(\gamma+1)}\left(Y_{(1)}-Y_{(n)}+\right.$ $2 a) / 2$, we have the asymptotic joint density of $\left(S^{\prime}, T^{\prime}\right)$ in a same manner to Subsection 2.1. So, the asymptotic expectation of ${S^{\prime}}^{2}$ is $E\left({S^{\prime}}^{2}\right)$ can be calculated. So, we may assume the following condition.

(B2) There exists a positive constant $B$ satisfying $E\left({S^{\prime}}^{2}\right) \rightarrow B$ as $n \rightarrow \infty$.

Concerning this assumption, we have the following lemma.

Lemma 2.2. (B2) and $E\left({S^{\prime}}^{4}\right)=O(1)$ hold under (A2).

The proof is omitted since it is similar to the one of Lemma 2.1.

Under the condition (B2), as $n \rightarrow \infty$,

$$
E\left(n^{2 /(\gamma+1)} M_{n}^{2}\right) \rightarrow B \xi^{2} .
$$

If $\theta$ is estimated by the midrange $M_{n}=\left(X_{(1)}+X_{(n)}\right) / 2$, then the risk is given by

$$
r_{n}=E\left(M_{n}-\theta\right)^{2}+d n,
$$

where $d(>0)$ is the cost per observation. From $S^{\prime}=n^{1 /(\gamma+1)}\left(M_{n}-\theta\right) / \xi$ and (2.9), $r_{n}$ is approximated by $B \xi^{2} n^{-2 /(\gamma+1)}+d n$, which is minimized at the 
integer closest to $n=n_{d}^{(2)}:=\left\{2 B \xi^{2} /(d(\gamma+1))\right\}^{(\gamma+1) /(\gamma+3)}$ and the minimized value is

$$
r_{n_{d}^{(2)}}=B \xi^{2}\left(\frac{d(\gamma+1)}{2 B \xi^{2}}\right)^{2 /(\gamma+3)}\left(\frac{\gamma+3}{\gamma+1}\right) .
$$

However, unless $\xi$ is known, one can not attain this risk with a non-sequential procedure. Since the range $R_{n}=X_{(n)}-X_{(1)}$ converges to $2 a \xi$ almost surely as $n \rightarrow \infty$, therefore we consider the following stopping rule:

$$
T_{d}^{(2)}:=\inf \left\{n \geq m_{d}^{(2)} \mid n^{(\gamma+3) /(\gamma+1)} \geq B R_{n}^{2} /\left(2 a^{2} d(\gamma+1)\right)\right\},
$$

where $m_{d}^{(2)}$ is the initial sample size with $d^{-l} \leq m_{d}^{(2)}=o\left(d^{-(\gamma+1) /(\gamma+3)}\right)(0<$ $l<(\gamma+1) /(\gamma+3))$. Then we have the following theorem.

Theorem 2.2. Under the conditions (A2) and (B2), as $d \rightarrow 0$, we have
(i) $T_{d}^{(2)} / n_{d}^{(2)} \stackrel{\text { a.s. }}{\rightarrow} 1$,
(ii) $E\left(T_{d}^{(2)}\right) / n_{d}^{(2)} \rightarrow 1$,
(iii) $r_{T_{d}^{(2)}} / r_{n_{d}^{(2)}} \rightarrow 1$

The proof is omitted since it is similar to the one of Theorem 2.1.

From Theorem 2.2 and Chow and $\mathrm{Yu}$ (1981), as $d \rightarrow 0$,

$$
\frac{r_{T_{d}^{(2)}}}{r_{T_{d}^{\prime}}^{\prime}} \approx \frac{\left(\frac{d(\gamma+1)}{2 B \xi^{2}}\right)^{2 /(\gamma+3)}\left(1+\frac{2 B \xi^{2}}{\gamma+1}\right)}{2 \sqrt{d} \sigma} \rightarrow \begin{cases}0 & (0<\gamma<1) \\ \text { constant } & (\gamma=1), \\ \infty & (\gamma>1),\end{cases}
$$

where $\sigma^{2}=V\left(X_{1}\right)$. So, the estimation procedure $\left(T_{d}^{(2)}, M_{T_{d}^{(2)}}\right)$ is asymptotically better than $\left(T_{d}^{\prime}, \bar{X}_{T_{d}^{\prime}}\right)$ for $0<\gamma<1$, and worse for $\gamma>1$. In other words, $\left(T_{d}^{(2)}, M_{T_{d}^{(2)}}\right)$ is asymptotically superior to $\left(T_{d}^{\prime}, \bar{X}_{T_{d}^{\prime}}\right)$ if the density changes sharply at the end points of the support. Koike (2007) observed a similar asymptotic superiority of the sequential estimation procedure based on the midrange in the sequential interval estimation procedure for $\theta$ under the same assumptions when the density changes steeply at the end points of the support. Note that similar results for the location family in the nonsequential case can be found in Akahira (1975a) and Akahira and Takeuchi (1981, 1995).

\section{ACKNOWLEDGMENTS}

The author would like to thank Professor Masafumi Akahira of University of Tsukuba for his valuable comments throughout the preparation of the paper. 
The author also thanks the Editor and the referee for helpful comments which have led to improvements to the original version of the manuscript. This research was partly supported by the Grant-in-Aid for Scientific Research (C) 17540101, Japan Society for the Promotion of Science.

\section{REFERENCES}

Akahira, M. (1975a). Asymptotic Theory for Estimation of Location in Non-Regular Cases, I: Order of Convergence of Consistent Estimators, Reports of Statistical Application Research, Union of Japanese Scientists and Engineers 22: 8-26.

Akahira, M. (1975b). Asymptotic Theory for Estimation of Location in Non-Regular Cases, II: Bounds of Asymptotic Distributions of Consistent Estimators, Reports of Statistical Application Research, Union of Japanese Scientists and Engineers 22: 99-115.

Akahira, M. and Koike, K. (2005). Sequential Interval Estimation of a Location Parameter with the Fixed Width in the Uniform Distribution with an Unknown Scale Parameter, Sequential Analysis 24: 63-75.

Akahira, M. and Takeuchi, K. (1981). Asymptotic Efficiency of Statistical Estimators: Concepts and Higher Order Asymptotic Efficiency, Lecture Notes in Statistics 7, New York: Springer-Verlag.

Akahira, M. and Takeuchi, K. (1995). Non-Regular Statistical Estimation, Lecture Notes in Statistics 10\%, New York: Springer-Verlag.

Akahira, M. and Takeuchi, K. (2003). The Information Inequality in Sequential Estimation for the Uniform Case, Sequential Analysis 22: 223232.

Basawa, I. V., McCormick, W. P., and Sriram, T. N. (1990). Sequential Estimation for Dependent Observations with an Application to NonStandard Autoregressive Processes, Stochastic Processes and Their Applications 35: 149-168.

Chaturvedi, A., Surinder, K., and Sanjeev, K. (2001). Multi-Stage Estimation Procedures for the "Range" of Two-Parameter Uniform Distribution, Metron 59: 179-186.

Chow, Y. S. and Martinsek, A. T. (1982). Bounded Regret of a Sequential Procedure for Estimation of the Mean, Annals of Statistics 10: 909-914.

Chow, Y. S. and Yu, K. F. (1981). On the Performance of Sequential Procedure for the Estimation of the Mean, Annals of Statistics 9: 184189.

Ghosh, M. and Mukhopadhyay, N. (1979). Sequential Point Estimation of the Mean When the Distribution is Unspecified, Communications in Statistics-Theory \&5 Methods 8: 637-652. 
Ghosh, M., Mukhopadhyay, N., and Sen, P. K. (1997). Sequential Estimation, New York: Wiley.

Govindarajulu, Z. (1997). A Note on Two-Stage and Sequential FixedWidth Intervals for the Parameter in the Uniform Density, Statistics \& Probability Letters 36: 179-188. Erratum: Statistics \& Probability Letters 42 (1999): 213-215.

Koike, K. (2007). Sequential Interval Estimation of a Location Parameter with the Fixed Width in the Non-Regular Case, Sequential Analysis 26: $63-70$.

Lai, T. L. (1996). On Uniform Integrability and Asymptotically RiskEfficient Sequential Estimation, Sequential Analysis 15: 237-251.

Mukhopadhyay, N. (1987). A Note on Estimating the Range of a Uniform Distribution, South African Statistical Journal 21: 27-38.

Mukhopadhyay, N., Ghosh, M., Hamdy, H. I., and Wackerly, D. D. (1983). Sequential and Two-Stage Point Estimation for the Range in a Power Family Distribution, Sequential Analysis 2: 259-288.

Robbins, H. (1959). Sequential Estimation of the Mean of a Normal Population, in Probability and Statistics (Harold Cramér Volume), U. Grenander, ed., pp. 235-245, Stockholm: Almquist and Wiksell.

Serfling, R. J. (1970). Moment Inequalities for the Maximum Cumulative Sum, Annals of Mathematical Statistics 41: 1227-1234.

Wald, A. (1950). Statistical Decision Function, New York: Wiley. 